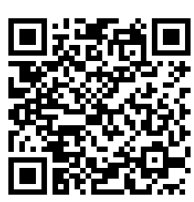

\section{Article Review "Sensorimotor Criteria for the Formation of the Autonomic Overstrain of the Athletes' Cardiovascular System", authors Romanchuk O. P., Guzii O. V.}

\author{
Polianska O. S. (Reviewer) ${ }^{1}$ \\ ${ }^{1}$ Bukovinian State Medical University, Ukraine
}

Received: 14.08.2020; Accepted: 15.09.2020; Published: 20.12.2020

\section{Review:}

The paper of Romanchuk and Guzii on "Sensorimotor criteria for the formation of the autonomic overstrain of the athletes' cardiovascular system" has been published in the International Journal of Science Annals, Vol. 3, No. 1, 2020.

The problem of diagnosticating states of fatigue, cardiovascular overstrain and overtraining is important in the practice of current athletes examinations.

This allows you to detect early signs of pathological states, which do not only limit the ability of sport perfection but also can lead to the development of critical states.

The authors introduce into the training process of highly qualified athletes methods of spiroarteriocardiorhythmography (SACR) and computerized motion meter (CCM), which are express multifunctional methods of studying the cardiorespiratory and sensorimotor systems and can be used in the "field".

Algorithm for determining the autonomic regulation overstrain of the cardiovascular system, based on the well-known method that was suggested by Shlyk, is especially noteworthy.

Based on determining the type of the heart rate autonomic regulation before, after and the next morning after training, the authors identified options that clearly characterize the autonomic regulation overstrain by sympathetic and parasympathetic type.

It is shown that such options occur in about $10 \%$ of cases among highly qualified athletes.
Namely, they are found in 19 out of 202 studied people. Of course, it would be appropriate to conduct other instrumental, hematological and biochemical studies that would characterize the proper humoral status of athletes.

However, it should be recalled that the studies are conducted in the "field", when the use of any invasive research methods is extremely limited.

Taking into account the aim of the work, the authors analyzes the indicators of sensorimotor function, which are obtained simultaneously with the determination of autonomic regulation of heart rate.

The used device (computerized motion meter) is valid for determining the time of motor reactions, which has a strong enough confirmation in the scientific publications of other authors.

The authors analyze the indicators of switching central settings, on the importance of which the author focused in previous publications, out of the 28 indicators that characterize motor reactions.

Having used non-parametric methods of statistical analysis, the authors prove significant differences between these indicators, which are obtained while testing with the right and left hand.

It is shown that the dynamics of their changes in athletes with overstrain of an autonomic regulation of the cardiovascular system by sympathetic and parasympathetic type has a characteristic asymmetry, which, according to other authors, may indicate the peculiarities of ergotropic and trophotropic processes in the body. 
The latter is important in the diagnosticating of fatigue, overfatigue, overstrain, as well as overtraining. I think that further research in this direction is prospective for the early diagnosis of such states in the currently examinations of athletes.

Despite the fact that the authors do not divide athletes with consideration to kind of sport, which is certainly important for the formation of sensorimotor reactions, it should be noted the importance of this work, the results of which show the relationship between autonomic regulation of the cardiovascular system and the mechanisms of the central movement control.

The paper is written in a scientific language, and at the same time, it is not overloaded with highly specialized terminology.
It would be appropriate to use newer literary sources. However, it is understandable that the authors wish to focus on the significant results obtained by using other modern methods, as well as the results obtained when using this device in earlier studies.

The paper is compiled on a high scientific level and is of high theoretical and practical interest. The obtained results can be used by doctors of sports medicine and coaches.

Reference
Romanchuk, O. P., \& Guzii, O. V. (2020).
Sensorimotor criteria for the formation of the autonomic
overstrain of the athletes' cardiovascular system.
International Journal of Science Annals, 3(1), 46-53.
doi:10.26697/ijsa.2020.1.6

\section{Cite this article as:}

Polianska, O. S. (2020). Article Review "Sensorimotor criteria for the formation of the autonomic overstrain of the athletes' cardiovascular system”, authors Romanchuk O. P., Guzii O. V. International Journal of Science Annals, 3(2), 39-40. doi:10.26697/ijsa.2020.2.7

The electronic version of this article is complete. It can be found online in the IJSA Archive https://ijsa.culturehealth.org/en/arhiv and in the KRPOCH Publishing Repository https://ekrpoch.culturehealth.org/handle/lib/71 This is an Open Access article distributed under the terms of the Creative Commons Attribution License, which permits unrestricted use, distribution, and reproduction in any medium, provided the original work is properly cited (http://creativecommons.org/licenses/by/4.0/deed.en). 